\title{
Algoritmo Híbrido Genético-Fuzzy Aplicado no Projeto de Amplificadores Raman
}

\author{
Gustavo C. M. Ferreira, M. H. M. Paiva, M. J. Pontes e M. E. V. Segatto * \\ Márcio Freitas **
}

\begin{abstract}
Resumo-Este artigo propõe a utilização de um algoritmo híbrido genético-fuzzy combinado com um modelo analítico para o projeto de amplificadores Raman multi-bombeios. O objetivo é encontrar uma combinação de parâmetros capaz de atender especificações de projeto. Como resultados do algoritmo obtémse os comprimentos de onda e as potências dos $\mathrm{N}$ bombeios analisados, assim como o ganho on-off e o ripple. O método proposto se mostrou robusto na análise simultânea de múltiplos parâmetros, como o número de bombeios, comprimentos de onda e potências nas quais os lasers de bombeio serão alocados.
\end{abstract}

Palavras-Chave-amplificadores ópticos, espalhamento Raman, otimização, algoritmos genéticos, lógica fuzzy.

Abstract-This paper proposes the use of a hybrid geneticfuzzy algorithm combined with an analytical model for the design of multi-pump Raman amplifiers. The goal is to find a combination of parameters able to meet design specifications. As a result of the algorithm the wavelength and the powers of $\mathrm{N}$ pumps analyzed are obtained, as well as on-off gain and ripple. The proposed method has shown to be robust in the simultaneous analysis of multiple parameters, such as the number of pumps, wavelength and power of lasers in which the pump will be allocated.

Keywords - optical amplifiers, Raman scattering, optimization, genetic algorithms, fuzzy logic.

\section{INTRODUÇÃO}

A demanda por redes de comunicação com capacidade cada vez mais elevadas continua a ser impulsionada pela expansão da Internet. A exploração de novos serviços resulta em um número cada vez maior de usuários, exigindo assim uma largura de banda crescente para a transmissão de múltiplos serviços. Sendo assim, faz-se necessário desenvolver redes de alta capacidade para prover a infra-estrutura exigida. As redes ópticas foram desenvolvidas para suprir tais necessidades, uma vez que as fibras ópticas possibilitam a transmissão de sinais digitais de dezenas de $\mathrm{Gb} / \mathrm{s}$ em longas distâncias, além de serem pouco suscetíveis às interferências eletromagnéticas [1].

Para superar limitações devido principalmente à atenuação das fibras nos enlaces de longa distância e permitir altas taxas de transmissão, com dispositivos transparentes ao aumento das taxas de bits, surgiram os amplificadores ópticos. Estes amplificadores permitiram a amplificação simultânea de múltiplos canais e a implementação das redes multi-comprimento de onda ou redes ópticas WDM (Wavelength Division Multiplexing).

*Laboratório de Telecomunicações (http://www.labtel.ele.ufes.br). Universidade Federal do Espírito Santo, Vitória, ES. Contato: gmariotto@gmail.com ${ }^{* *}$ Optiwave Corp. Inc., Ottawa, Canada. Trabalho parcialmente fianciado pelo CNPq, projeto número 309049/2006-7.
Os amplificadores ópticos do tipo EDFA (Erbium Doped Fiber Amplifiers) foram rapidamente desenvolvidos e implementados comercialmente em larga escala. Já os amplificadores Raman foram desenvolvidos posteriormente, sendo atualmente requisitados em muitas aplicações por apresentarem uma flexibilidade maior na escolha da banda de sinais, além da possibilidade de serem implantados em enlaces de fibras já instaladas. Outra característica marcante é o fato destes amplificadores apresentarem uma relação sinalruido óptica (OSNR) superior à apresentada pelos EDFAs. Os amplificadores Raman também podem ser implementados em um enlace híbrido com os EDFAs.

Os principais parâmetros a serem definidos no projeto de um amplificador Raman multi-bombeios são os comprimentos de onda e as potências dos bombeios que, combinados, forneçam os valores de ganho on-off e ripple definidos a priori. Portanto, a otimização de amplificadores Raman é um problema multiobjetivo, o que exige métodos robustos para encontrar boas soluções.

A otimização de amplificadores Raman através da utilização de algoritmos robustos e elaborados é apresentada em [2], [3], [4], [5], [6]. Por demonstrar que o problema de design do amplificador pode ser quebrado em duas partes mais simples [2], acarretou em um grande avanço na busca de resultados ótimos. A base do algoritmo é baseada em soluções numéricas do sistema completo das equações de propagação acopladas. O procedimento em [2] possibilitou um decréscimo no ripple de uma ordem de magnitude maior do que um. Porém, o método apresentado em [2] é a convergência relativamente lenta do algoritmo genético implementado. Para superar tal dificuldade, [4] propõe um método que aproxima a curva de coeficiente de ganho Raman utilizando diversas linhas assintóticas, chamado de técnica de compensação geométrica.

Um algoritmo mais robusto foi apresentado em [5], combinando compensação geométrica com técnicas de algoritmos genéticos-híbridos [3]. Em [5], Liu et. al mostrou-se que o tempo de processamento da simulação com o algoritmo genético-híbrido pode ser reduzido quando combinado com compensação geométrica.

Neste artigo é proposto um sistema híbrido genético-fuzzy capaz de encontrar boas soluções, para diversas configurações dos amplificadores, sem a necessidade de uma análise prévia do espaço de soluções. A importância da escolha correta dos parâmetros dos bombeios se deve a estes serem responsáveis pela configuração do ganho, que é uma das principais características de qualidade do amplificador.

O artigo está organizado como segue. Nas duas próximas seções, são descritas as bases teóricas deste trabalho: na seção 
II, as principais características dos amplificadores Raman e na seção III, as ferramentas computacionais utilizadas. $\mathrm{Na}$ seção IV é discutida a metodologia adotada, e os resultados obtidos são apresentados na seção V. Nossas conclusões são comentadas na seção VI.

\section{AMPLIFICADORES RAMAN}

Os amplificadores Raman baseiam-se no fenômeno de espalhamento Raman. Consistem em um ou múltiplos lasers de bombeio operando em alta potência, além da fibra óptica e dispositivos em fibra passivos tais como acopladores e despolarizadores. Para tais lasers de bombeio, a luz espalhada cresce rapidamente, com boa parte da energia do bombeio sendo convertida em luz espalhada. Com a alocação correta dos comprimentos de onda dos lasers de bombeio, é possível aproveitar a energia fornecida pelo bombeio, a qual é convertida em luz espalhada, para amplificar os sinais ou canais de informação.

\section{A. Esquemas de Bombeamento}

Há três esquemas básicos de bombeamento para os amplificadores Raman, conhecidos como co-propagante, contrapropagante e bi-direcional [7].

Neste trabalho são considerados amplificadores com dois a cinco lasers de bombeio contra-propagantes, como o mostrado na Fig. 1.

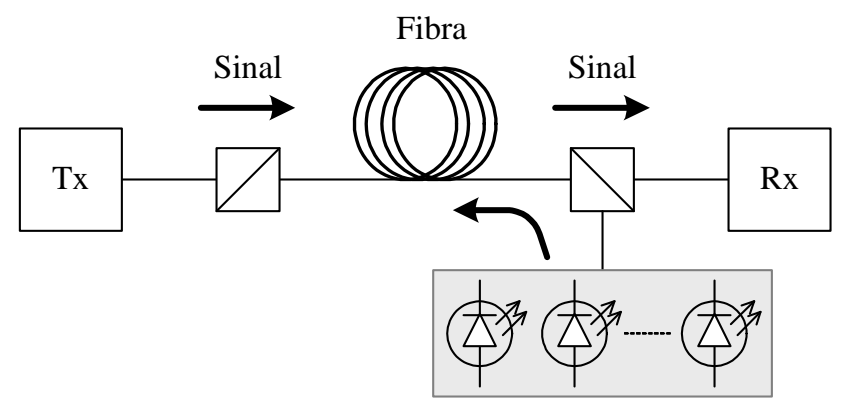

Bombeio

Fig. 1. Configuração contra-propagante.

A decisão pelo esquema contra-propagante se deve a que tal configuração é menos susceptível a efeitos não lineares e garante um ganho alto e razoável OSNR.

\section{B. Tipos de Amplificadores Raman}

Em termos de topologia, há dois tipos de amplificadores Raman, amplificadores Raman distribuídos e amplificadores Raman discretos.

O amplificador distribuído consiste em acoplar um ou mais lasers de bombeio de alta potência à própria fibra de transmissão do enlace. A principal vantagem desta topologia é apresentar valores de OSNR mais altos. Isto é resultado do sinal amplificado não oscilar entre valores muito baixos e nem muito altos, evitando, respectivamente, entrar em regiões de muito ruído e em regiões onde os efeitos não-lineares levariam a distorções no sinal.
Na topologia discreta, existe uma concentração da potência de bombeio e, consequentemente, a amplificação do sinal ocorre em uma região isolada da fibra. Neste caso, o sinal pode apresentar perdas muito grandes antes da amplificação e ganhos elevados após, sendo assim mais suscetível ao ruído e às não linearidades. A vantagem desta topologia está em apresentar melhor conversão de potência do que a distribuída, já que sua amplificação é obtida, em geral, em fibras de maior ganho. Geralmente amplificadores discretos são utilizados em sistemas utilizando fibras compensadoras de dispersão, concentrando-se o bombeio dentro destas fibras.

Nas análises feitas para este trabalho, é utilizado o amplificador Raman Distribuído, implementado em enlaces de fibras monomodo padrão (SMF). A vantagem de tal escolha é a possibilidade de utilizar somente um tipo de fibra durante todo o sistema e evitar tanto as regiões de alto ruído quanto os efeitos não-lineares.

\section{Modelo Analítico}

Para a solução do conjunto de equações que descrevem o funcionamento do efeito Raman, responsável pela amplificação dos sinais, é utilizado um modelo analítico descrito e validado em [8].

A evolução das potências e do ganho dos sinais é calculada para sistemas com múltiplos lasers de bombeio e múltiplos sinais. Leva-se em consideração a interação entre bombeios e sinais devido ao efeito Raman, os efeitos de polarização, desconsiderando, porém, efeitos de ruído tais como emissão espontânea amplificada e espalhamento de Rayleigh, e a saturação do ganho. Este modelo tem como grande vantagem a velocidade de cálculo, e é indicado em aplicações como a utilizada neste trabalho, que consiste na otimização simultânea (multi-objetivo) de dois parâmetros do amplificador Raman através do ajuste dos comprimentos de onda e das potências dos lasers de bombeio.

Após um processo de iteração entre frequências de bombeio, descrito em [9], pode-se obter a seguinte equação analítica genérica que descreve a evolução da potência dos bombeios ao longo da fibra:

$$
\begin{aligned}
& P_{\rho}(z)=P_{\rho}(\mathrm{L}) \exp [-\alpha(\mathrm{L}-z)] \\
& \exp \left[\sum_{\psi>\rho}\left[\mathrm{A}(\rho, \psi) \frac{1-\exp [\Lambda(z) \mathrm{B}(\psi, \varphi)]}{\mathrm{B}(\psi, \varphi)}\right]\right] \\
& \exp \left[\sum_{\psi<\rho}\left[\frac{-\rho}{\psi} \mathrm{A}(\rho, \psi) \frac{1-\exp [\Lambda(z) \mathrm{B}(\psi, \varphi)]}{\mathrm{B}(\psi, \varphi)}\right]\right],
\end{aligned}
$$

onde

$$
\begin{gathered}
\mathrm{A}(\rho, \psi)=\mathrm{C}_{\mathrm{R}, \rho \psi} \mathrm{P}_{\psi}(\mathrm{L}), \\
\Lambda(z)=-(1-\exp [-\alpha(\mathrm{L}-z)]) /(\Gamma \alpha)
\end{gathered}
$$

$\mathrm{e}$

$$
\mathrm{B}(\psi, \varphi)=\sum_{\varphi<\psi} \psi \mathrm{C}_{R, \psi \varphi} \mathrm{P}_{\varphi}(\mathrm{L}) / \varphi-\sum_{\varphi>\psi} \mathrm{C}_{\mathrm{R}, \psi \varphi} \mathrm{P}_{\varphi}(\mathrm{L}) .
$$

Nas expressões acima, L é o comprimento da fibra, $\mathrm{P}$ é a potência dos bombeios nas frequências ópticas $\rho, \psi$ e $\varphi, \alpha$ é o coeficiente de atenuação da fibra e $C_{R, x y}$ é o coeficiente de ganho de Raman entre as frequências $x$ e $y$. 
Com essa equação de evolução de potência dos bombeios pode-se demonstrar, como descrito em [9], que o ganho on-off dos sinais transmitidos pode ser calculado em dB por:

$$
\mathrm{G}_{\text {on-off }}(\nu, \mathrm{L})=10 \log \left(\exp \left[\int_{0}^{\mathrm{L}}\left(\sum_{N} \frac{\mathrm{C}_{\mathrm{R}, \rho \nu}}{\Gamma} \mathrm{P}_{\rho}(z) \mathrm{d} z\right)\right]\right),
$$

onde $v$ representa as frequências ópticas dos sinais e $\mathrm{N}$ é o número total de bombeios.

A solução de (1) e (2) permite analisar o espaço de soluções para valores de ripple e ganho on-off obtidos para as diferentes configurações de amplificadores Raman multi-bombeios.

\section{FERRAMENTAS}

Este trabalho visa a otimização de amplificadores Raman através do uso de algoritmos capazes de encontrar boas soluções para problemas multi-objetivo. Pela eficácia na busca de soluções dentro de espaços amostrais de difícil descrição, optou-se pela utilização de algoritmo genético, que ficaria responsável pela otimização dentro do espaço de soluções.

Posteriormente, devido à necessidade de uma abrangência maior para o código genético, e pela facilidade de se trabalhar com sistemas fuzzy para se definir um processo de decisão, decidiu-se trabalhar com um algoritmo híbrido genético-fuzzy.

\section{A. Algoritmos Genéticos}

Os algoritmos genéticos são algoritmos de otimização que envolvem uma solução numérica, inspirados pelo processo de seleção natural e genética [10].

Um algoritmo genético é composto tipicamente por uma população inicial de soluções, uma forma de calcular a qualidade de cada solução individual (função fitness), um método de misturar fragmentos de soluções para formar novas soluções (crossover) e um operador de mutação para evitar a perda de diversidade dentro do grupo de soluções.

Os métodos de reprodução consistem na seleção aleatória de pares de indivíduos para sofrerem, ou não, os processos de crossover e mutação, necessários para a evolução do algoritmo. Neste caso, a escolha de cada indivíduo de um par é feita de forma aleatória de modo que a probabilidade de seleção esteja diretamente relacionada ao seu valor de fitness. Logo, o melhor indivíduo tem maiores chances de ser selecionado.

Já o processo de crossover baseia-se na probabilidade de cruzamento. Cada par de indivíduos selecionados na reprodução tem uma probabilidade pré-definida de realizar um cruzamento. No caso de ocorrer cruzamento, será definido, aleatoriamente, um ponto dos indivíduos onde ocorrerá o cruzamento de informações, passando para a próxima geração dois novos indivíduos, resultantes desse cruzamento.

No caso da mutação, os indivíduos gerados pelo crossover terão uma probabilidade pré-definida de que um dos bits do indivíduo analisado seja invertido. Isso evita a perda de variabilidade do algoritmo.

Após a realização destes processos, sua população passa por um processo onde a resposta de cada indivíduo é analisada, de forma a gerar um valor correspondente de fitness, que consiste basicamente em definir a qualidade da resposta em uma escala de zero a um, onde o valor um indicaria a melhor resposta obtida dentro de determinadas expectativas.

Tais processos são realizados repetidamente até que algum parâmetro de fitness seja atingido, ou se atinja um número máximo de gerações.

\section{B. Sistemas fuzzy}

Um sistema baseado em regras fuzzy, ou simplesmente um sistema fuzzy, é uma ferramenta matemática desenvolvida a partir de conceitos da lógica fuzzy, que pode ser vista como uma extensão da lógica binária.

Existem quatro componentes fundamentais que caracterizam um sistema baseado em regras fuzzy: um processador de entrada, uma base de conhecimento, uma máquina de inferência, e um processador de saída [11].

Inicialmente, cada variável real do sistema é modelada por meio de conjuntos fuzzy, tantos quantos sejam necessários para identificar os diversos níveis que a variável pode assumir. A tarefa do processador de entrada é mapear cada valor de entrada em algum ponto do conjunto fuzzy apropriado.

Os conjuntos fuzzy que caracterizam as variáveis de entrada e de saída do sistema são armazenados em uma base de conhecimento, que contém ainda as regras para determinar o comportamento do sistema. Neste contexto, uma regra é uma proposição condicional do tipo $\mathrm{Se}<$ antecedente $>$ Então $<$ consequente $>$. A base de conhecimento dá suporte à ação da máquina de inferência, que é a responsável pelo controle de todo o processo.

De modo geral, a saída do processo de inferência é um conjunto fuzzy. Sendo assim, é necessário escolher um número real que represente bem a saída do sistema. Esse procedimento é realizado pelo processador de saída, de acordo com algum método adequado à aplicação em questão.

\section{Algoritmo Híbrido Genético-Fuzzy}

Existem duas maneiras de integrar algoritmos genéticos com lógica fuzzy. Pode-se aplicar técnicas de algoritmos genéticos para ajustar parâmetros de sistemas fuzzy, ou ainda utilizar a lógica fuzzy em algum dos processos do algoritmo genético [12].

Por exemplo, pode-se utilizar sistemas fuzzy com o objetivo de classificar os indivíduos gerados pelo algoritmo genético, o que geralmente é feito pela função fitness. Isso é particularmente útil quando o espaço de soluções é variável com relação aos parâmetros de entrada do sistema.

Em sistemas híbridos, busca-se uma combinação dessas duas técnicas, onde o algoritmo genético é um componente dinâmico, que realiza uma busca em um espaço de soluções, enquanto o sistema fuzzy é um componente estático, que define a relação e grau de influência dos dados de cada indivíduo, classificando-o.

Considerando as duas técnicas, é possível obter um algoritmo mais abrangente, capaz de lidar com uma diversidade maior de casos sem a necessidade de análise prévia do sistema. 


\section{Metodologia}

O objetivo deste trabalho é desenvolver uma metodologia de projeto de amplificadores Raman que possuam máximo ganho e mínimo ripple. Nossa metologia emprega o modelo analítico descrito em [8] e [9] para determinar o ganho on-off e ripple do amplificador e um algoritmo híbrido genético-fuzzy para avaliar e escolher novas topologias do amplificador. Em geral, partimos do número $\mathrm{N}$ de bombeios, nível de potência mínima e máxima de cada bombeio, faixa de comprimentos de onda de cada laser de bombeio e banda de amplificação desejada.

Para exemplificar a importância do processo de otimização faremos uma breve análise de um amplificador Raman com dois $(\mathrm{N}=2)$ bombeios na configuração contra-propagante, 100 quilômetros de fibra monomodo padrão e operando na Banda C.

A Fig. 2 mostra curvas de ganho para dois casos distintos. No Caso 1, os comprimentos de onda e as potências dos bombeios são, respectivamente, 1423, $2 \mathrm{~nm}$ e 1457, $2 \mathrm{~nm}$, com 113, $1 \mathrm{~mW}$ e 293, $0 \mathrm{~mW}$. Já no Caso 2 considera-se os comprimentos de onda de bombeio iguais a 1414, $1 \mathrm{~nm}$ e 1416,7 $\mathrm{nm}$, com respectivas potências de $247,7 \mathrm{~mW}$ e 263, $8 \mathrm{~mW}$.

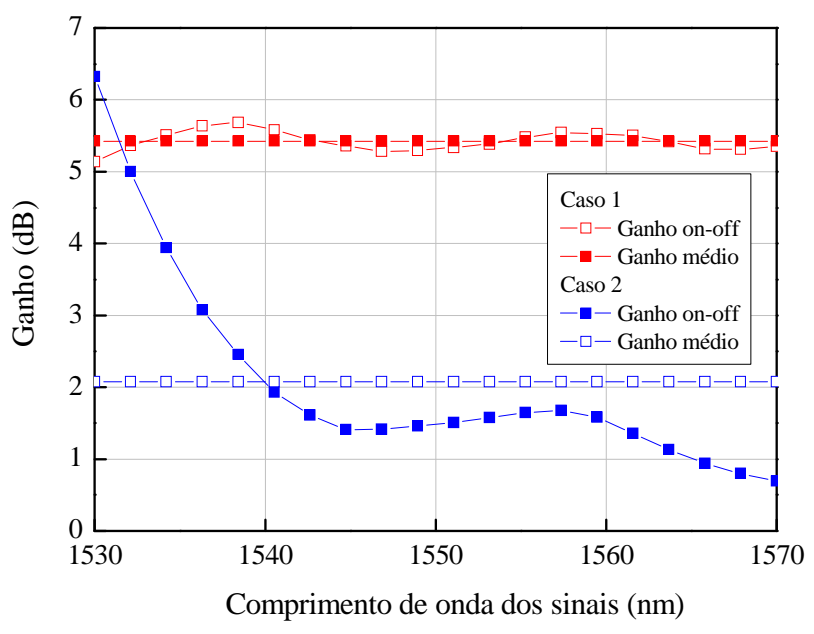

Fig. 2. Curvas de Ganho on-off em função do comprimento de onda dos sinais de entrada.

A diferença observada nos valores de ganho na Fig. 2 ilustra claramente a necessidade de se escolher de forma adequada os comprimentos de onda e as potências dos bombeios. Pode-se verificar que o Caso 2 apresenta uma variação muito grande de ganho e um ganho médio baixo, o que não é interessante para um projeto de amplificador, enquanto o Caso 1 apresenta valores mais adequados.

Devido à necessidade de se trabalhar com os objetivos de maximizar ganho e minimizar ripple decidiu-se utilizar algoritmos genéticos. Contudo, a escolha de utilizar algoritmos genéticos acarretava em um novo problema, que era como definir uma função fitness para um sistema onde não se conhece o seu espaço de soluções.

Para que fosse possível encontrar uma função que atendesse a tais requisitos, era necessário primeiro recriar o espaço de soluções através de uma nuvem de pontos e a partir daí, formular uma equação de fitness. Porém, ao mudarmos a configuração de dois para quatro bombeios, por exemplo, era necessário refazer todo o processo.

Para contornar esse problema, recorreu-se a um algoritmo híbrido genético-fuzzy, capaz de se adaptar a cada configuração e, ainda assim, manter a qualidade das respostas obtidas.
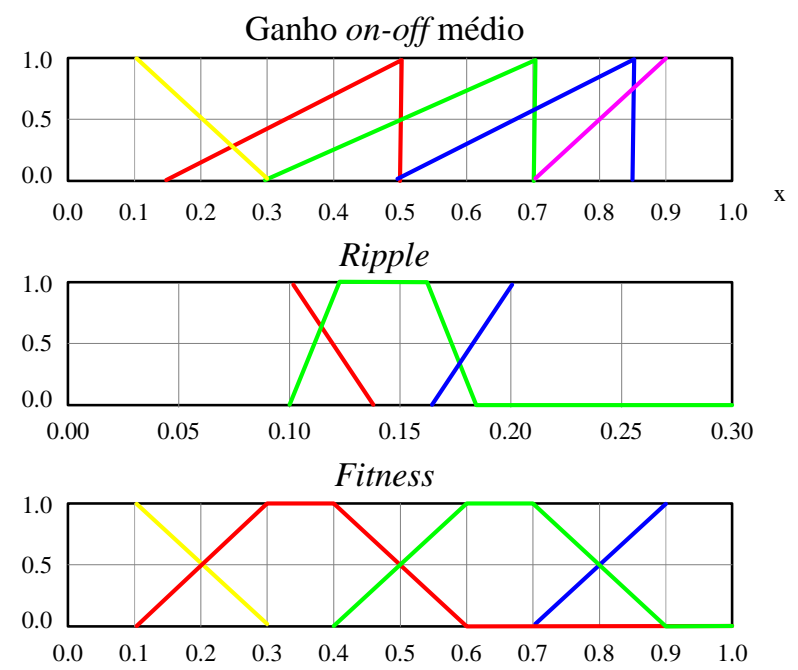

Fig. 3. Sistema fuzzy.

O sistema fuzzy é utilizado para atribuir um valor de fitness a cada indivíduo gerado pelo algoritmo genético, de acordo com os valores de ganho on-off médio e ripple do indivíduo.

A Figura 3 mostra os conjuntos fuzzy considerados para cada variável do sistema. Na parte superior da figura podem se observadas cinco curvas, que representam os níveis assumidos para a variável de entrada ganho on-off: 'ruim', 'baixo', 'regular', 'bom' e 'excelente'. No meio, ilustram-se as três curvas que definem a variável de entrada ripple: 'excelente', 'regular' e 'ruim'. E na parte inferior da figura, as quatro curvas caracterizam a variável de saída fitness como 'ruim', 'regular', 'bom' e 'excelente'.

É importante frisar que as curvas mostradas na Fig. 3 para as variáveis de entrada não são fixas. Elas devem ser ajustadas, uma vez que alterações na configuração do sistema, como é o caso do número de bombeios, geram alterações no domínio das variáveis de entrada. Os ajustes são feitos com base nos melhores valores encontrados em 100 configurações do amplificador Raman geradas aleatoriamente.

Combinando os cinco termos possíveis do ganho on-off e os três do ripple, cria-se um conjunto de 15 regras para modelar o sistema. Para exemplificar, uma das regras adotadas é: Se o ganho on-off é excelente, porém o ripple é ruim, então o fitness é ruim, já que buscamos respostas cujo ripple seja menor do que $1 \mathrm{~dB}$.

Todos os possíveis resultados de ganho on-off e ripple, para qualquer indivíduo do algoritmo genético, são processados pelo sistema fuzzy. Com esses pares de valores de entrada e seus respectivos valores de saída pode-se gerar uma tabela de fitness que sintetiza a ação do sistema fuzzy, e que pode ser consultada com facilidade, a fim de definir o valor de fitness para um determinado indivíduo. 
A saída do sistema fuzzy é um número pertencente ao intervalo $[0,1]$, que representa a qualidade do indivíduo. O fitness unitário indica que tanto o ganho on-off médio quanto o ripple se encontram na região da curva que os descreve como excelentes.

\section{Resultados}

Dessa forma, a partir da definição do número de bombeios a ser utilizado no amplificador, são definidos os valores limite para potência, e comprimento de onda para cada bombeio, e o número de gerações do algoritmo genético.

A partir dessas definições, o algoritmo genético utiliza um modelo analítico para verificar os valores de ganho on-off e ripple de cada um de seus indivíduos e, posteriormente, seu valor de fitness, através da tabela gerada pelo sistema fuzzy. Em seguida, o algoritmo genético inicia o processo de evolução através das gerações.

Nos experimentos realizados, limitou-se o comprimento de onda dos bombeios ao intervalo de $1410,0 \mathrm{~nm}$ a $1470,0 \mathrm{~nm}$, enquanto variou-se a potência de $100 \mathrm{~mW}$ a $300 \mathrm{~mW}$. Os valores limite da potência se devem aos lasers comerciais com custo competitivo estarem neste intervalo. Considerouse nas análises um enlace com $100 \mathrm{~km}$ de extensão, e 20 sinais de entrada, igualmente espaçados entre 1530,0 $\mathrm{nm}$ e 1570, $0 \mathrm{~nm}$.

Já o algoritmo genético teve como parâmetros os valores de 20 indivíduos por geração, um número máximo de 1000 gerações e probabilidades de crossover de $70 \%$ e mutação de $8,33 \%$.

A Fig. 4 mostra a evolução do fitness do algoritmo híbrido para o caso de dois bombeios. Nota-se que a evolução do fitness ocorre de forma rápida, atingindo com poucas iterações um valor de fitness máximo que sofrerá poucas variações.

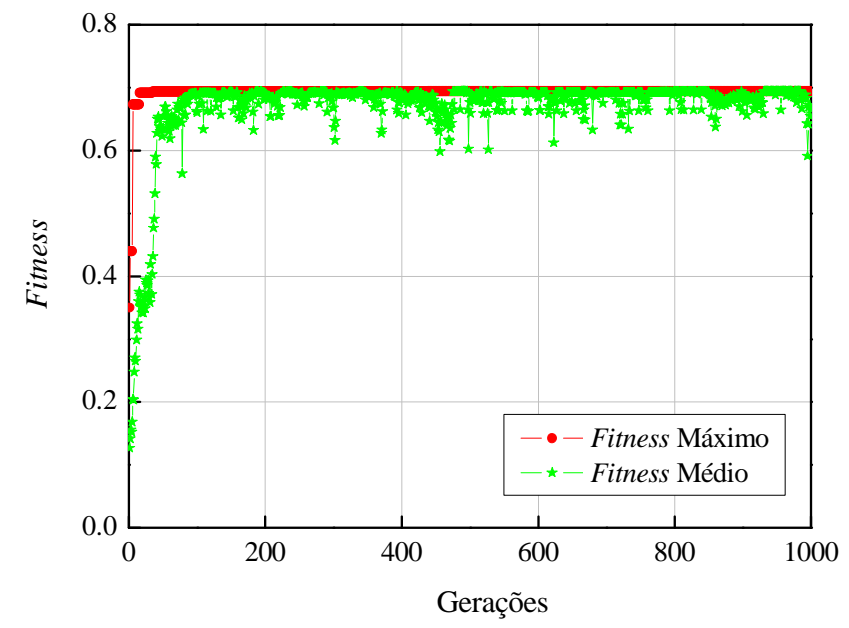

Fig. 4. Evolução da função fitness para o algoritmo híbrido com dois bombeios

Foram obtidos como parâmetros dos bombeios os valores de 1426, $9 \mathrm{~nm}$ e 1461, $6 \mathrm{~nm}$ para os comprimentos de onda, e $175,8 \mathrm{~mW}$ e $272,1 \mathrm{~mW}$ para as potências, respectivamente. Nesta configuração, é possível obter um ganho on-off médio de 5,7 dB e um ripple de $1 \mathrm{~dB}$, o que está dentro dos prérequisitos de projeto.

Na Fig. 4, a curva cheia representa o valor máximo de fitness obtido a cada geração, enquanto a curva contendo símbolos representa o valor médio dos fitness a cada geração. Pode-se observar que para o caso analisado, o indivíduo de melhor fitness foi mantido em todas as gerações futuras e que a média sofre variações bruscas, decorrentes dos processos de crossover e mutação utilizados no algoritmo genético.

Este procedimento foi repetido para configurações com três, quatro e cinco bombeios, com a intenção de verificar a funcionalidade do sistema fuzzy na obtenção de uma função fitness que fosse adequada para situações diversas.

A Figura 5 representa a evolução do fitness do algoritmo híbrido para o caso de cinco bombeios, que seria o outro extremo dos testes realizados. Neste caso, espera-se que o intervalo de convergência do algoritmo não seja muito variado, porém que o resultado obtido tenha um ganho on-off médio maior do que o obtido com dois bombeios, e um valor de ripple ainda menor ou igual a 1,0 dB.

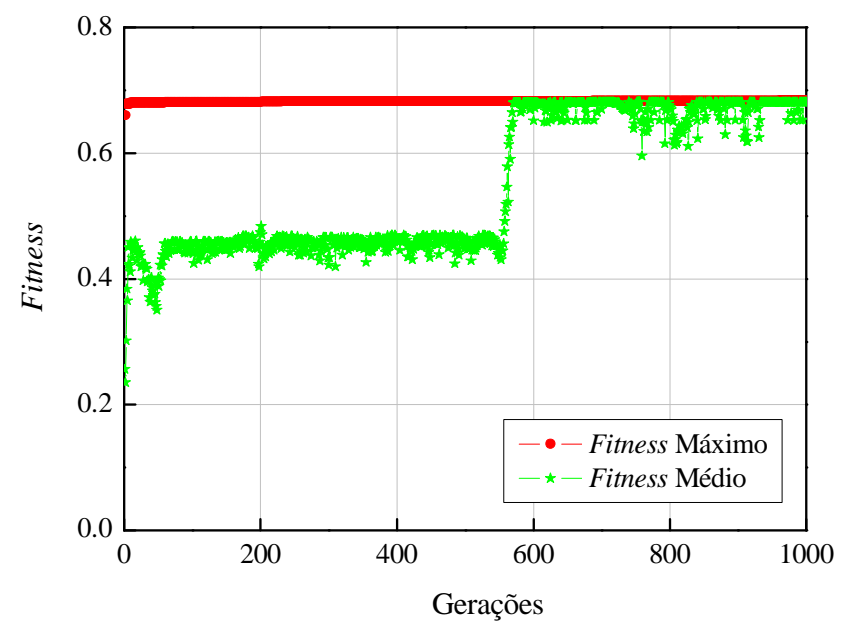

Fig. 5. Evolução da função fitness para o algoritmo híbrido com cinco bombeios.

É possível observar que tanto a Fig. 4 como a Fig. 5 têm uma curva de evolução de fitness muito semelhante. Em ambos os casos a convergência para valores de máximo ganho se dá em poucas gerações e o fitness máximo obtido fica próximo do mesmo valor.

O caso mostrado na Fig. 5 tem como parâmetros de bombeio os valores de comprimentos de onda 1415,0 $\mathrm{nm}$, 1423, $6 \mathrm{~nm}, 1432,0 \mathrm{~nm}, 1461,0 \mathrm{~nm}$ e $1465,9 \mathrm{~nm}$, e respectivas potências de bombeio de 281, $5 \mathrm{~mW}, 233,9 \mathrm{~mW}$, 128,5 mW, 225, $0 \mathrm{~mW}$ e 226, $4 \mathrm{~mW}$. Nesta configuração com cinco bombeios, obteve-se um ganho on-off médio de $12,2 \mathrm{~dB}$ e um ripple de $1 \mathrm{~dB}$.

Outro fator importante a ser mencionado é o tempo de processamento para se chegar aos resultados mostrados nas Fig. 4 e Fig. 5. Para o caso de 2 bombeios, foi necessário um tempo de aproximadamente quatro minutos para se chegar ao fim das iterações. Para 5 bombeios necessitou-se do dobro de tempo, o que demonstra que o aumento do número de 
bombeios resulta em maior tempo de processamento.

$\mathrm{Na}$ Tabela I apresentamos as respostas obtidas pelo algoritmo híbrido genético-fuzzy em comparação com as obtidas pelo algoritmo genético. Neste caso é analisada a configuração com cinco bombeios, porém com variação de comprimentos de onda de $1400 \mathrm{~nm}$ a $1500 \mathrm{~nm}$ e potências de $50 \mathrm{~mW}$ a $500 \mathrm{~mW}$.

TABELA I

COMPARAÇÃO DOS ALGORITMOS HÍBRIDO E GENÉTICO.

\begin{tabular}{c|c|c}
\hline & Híbrido & Genético \\
\hline & 1418,2 & 1410,9 \\
Comp. onda dos & 1421,7 & 1412,5 \\
bombeios [nm] & 1430,4 & 1424,9 \\
& 1451,9 & 1459,0 \\
& 1475,6 & 1459,6 \\
\hline & 444,2 & 417,5 \\
Potência dos & 260,0 & 155,2 \\
bombeios [mW] & 473,3 & 268,5 \\
& 474,0 & 304,9 \\
& 259,2 & 204,0 \\
\hline Ganho On-Off & 22,5 & 14,2 \\
médio [dB] & & \\
\hline ripple [dB] & 1 & 1 \\
\hline
\end{tabular}

A Tabela I mostra as diferenças entre os algoritmos híbrido e genético. No algoritmo genético é utilizada uma curva de fitness criada sem conhecimento prévio do espaço de soluções do problema e, sendo assim, a resposta ficou muito inferior àquela encontrada pelo algoritmo híbrido.

Fica possível mostrar pela Tabela I que a eficiência do algoritmo híbrido é maior que aquela apresentada pelo genético, já que o primeiro tem uma abrangência maior sem necessidade de mudanças no código.

\section{Conclusões}

Este trabalho analisou o problema da escolha dos lasers de bombeamento em um amplificador Raman, a partir de um algoritmo híbrido, onde um modelo analítico de solução para amplificadores Raman é utilizado. Algoritmos híbridos genético-fuzzy tem aplicações em diversas áreas. As potencialidades de cada método permitem gerar um algoritmo mais robusto e capaz de encontrar respostas melhores do que se forem usados separadamente.

Verificou-se a eficácia do algoritmo híbrido genético-fuzzy na busca de um método de otimização de amplificadores Raman. A utilização de tal método é crítica quando se leva em conta a necessidade de se aperfeiçoar uma função multiobjetivo.

A utilização de um sistema fuzzy como função fitness possibilita que um único código atenda a uma gama ampla de casos, sem a necessidade de realizar uma análise prévia do espaço de soluções.

Outro fator importante é a velocidade de processamento do algoritmo que, mesmo analisando um número grande de casos, consegue realizá-lo em um período de tempo consideravelmente curto, o que torna a solução ainda mais atraente.

\section{REFERÊNCIAS}

[1] R. Ramaswami and K. N. Sivarajan, Optical networks: a practical perspective, 2nd ed. London: Academic Press, 2001.
[2] V. E. Perlin and H. G. Winful, "Optimal design of flat-gain wide-band fiber raman amplifiers," IEEE J. Lightwave Technology, vol. 20, no. 2, p. 250, Feb. 2002.

[3] X. Liu and B. Lee, "Optimal design for ultra-broad-band amplifier," IEEE J. Lightwave Technology, vol. 21, no. 122, p. 3446, Dec. 2003.

[4] Z. Li, C. Liu, J. Chen, and C. Zhao, "A fast and stable method for raman amplifier propagation equations," Optics Express, vol. 12, no. 3, p. 436, 2004.

[5] X. Liu, J. Chen, C. Lu, and X. Zhou, "Optimizing gain profile and noise performance for distributed fiber raman amplifiers," Optics Express, vol. 12, no. 24, p. 6053, 2004.

[6] K. Fujimura, A. Oguri, T. Nakajima, Y. Emori, S. Namiki, and M. Sakano, "Applying a numerical simulation technique to the design of WDM-pumped raman amplifiers, and methods for the automatic determination of pump powers," Furukawa Review, vol. 25, no. 1, 2004.

[7] C. Headley and G. P. Agrawal, Raman amplification in fiber-optical communication systems. New York: Academic Press, 2005.

[8] S. P. N. Cani, "Contribuição ao modelamento analítico em amplificadores Raman," Doutorado em Engenharia Elétrica, Centro Tecnológico, Universidade Federal do Espírito Santo, Vitória, 2007.

[9] S. Cani, L. de Calazans Calmon, M. Pontes, M. Ribeiro, M. Segatto, and A. Cartaxo, "An analytical approximated solution for the gain of broadband Raman amplifiers with multiple counter-pumps," IEEE Journal of Lightwave Technology, vol. 27, no. 7, pp. 944-951, Abril 2009.

[10] D. A. Coley, An introduction to genetic algorithms for scientists and engineers. London: World Scientific, 1999.

[11] R. S. M. Jafelice, L. C. Barros, and R. C. Bassanezi, Teoria dos conjuntos fuzzy com aplicações. São Paulo: Editora Plêiade, 2005.

[12] F. Herrera and M. Lozano, "Fuzzy Genetic Algorithms: issues and models," University of Granada, Tech. Rep., 1999. 